\title{
SUMMABILITY METHODS BASED ON THE RIEMANN ZETA FUNCTION
}

\author{
LARRY K. CHU \\ DEPARTMENT OF MATHEMATICS AND COMPUTER SCIENCE \\ STATE UNIVERSITY OF NORTH DAKOTA - MINOT \\ MINOT, ND 58701 \\ (Received September 12, 1986)
}

ABSTRACT. This paper is a study of summability methods that are based on the Riemann Zeta function. A limitation theorem is proved which gives a necessary condition for a sequence $x$ to be zeta summable. A zeta summability matrix $Z_{t}$ associated with a real sequence $t$ is introduced; a necessary and sufficient condition on the sequence $t$ such that $Z_{t}$ maps $l_{1}$ to $l_{1}$ is established. Results comparing the strength of the zeta method to that of well-known summability methods are also investigated.

KEY WORDS AND PHRASES. Zeta summability method, zeta matrix method, $H$ matrix, Cesaro method, Euler-Knopp method.

1980 AMS SUBJECT CLASSIFICATION CODE. 40C05, 40C15, 40D05, 40D25.

1. INTRODUCTION.

Recall that the Riemann zeta function is given by $\quad s(s)=\sum_{k=1}^{\infty}\left(1 / k^{s}\right)$ lor $s>1 \quad$ (Titschmarch [1]). A number sequence is said to be zeta summable to $L$ (or $\zeta$-summable to $L$ ) provided that

$$
\lim _{s \rightarrow:^{+}} \frac{1}{s(s)} \sum_{n=1}^{\infty} \frac{x_{n}}{n^{s}}=L .
$$

The zeta method is a "sequence-to-function" summability method whose domain consists of those sequence $x$ such that the Dirichlet's series $\sum_{k=1}^{\infty}\left(x_{k} / k^{s}\right) \quad$ is convergent for $s>1$.

In the second section it is shown that the zeta summability method is regular and totally regular (preserves finite and infinite limits). A limitation theorem is proved which gives a necessary condition for a sequence $x$ to be zeta summable. In section 3 we introduce a zeta summability matrix $Z_{t}$ associated with a real sequence $t$; a necessary and sufficient condition on the sequence $t$ such that $Z_{t}$ maps $I_{1}$ into $I_{1}$ is established. The final section contains results comparing the strength of the zeta method to that of well-known summability methods. For example, the zeta method is stronger than the Cesàro method of order 1 but does not include the Cesàro method of order 2; the zeta method does not include and is not included in the Euler-Knopp method of order $r$ for $0<r<1$.

\section{BASIC THEOREMS}

THEOREM 1. The $\zeta$-summability method is totally regular.

Proof. First let $x$ be a sequence satisfying $\lim _{k} x_{k}=L$, and suppose $\varepsilon>0$. Then choose $N_{1}$ so that $k>N_{1}$ implies $\left|x_{k}-L\right|<\varepsilon / 2$. Now for any positive integer $k$ and $s>1$ we see that $\sum_{-h}^{N} N_{1}\left(\left|x_{h} \quad i,\right| / h^{s}\right)$ is 
bounded by $\sum_{k=1}^{N_{1}}\left|x_{k} \cdot L\right|=M$. Since $\quad \sum_{k=1}^{\infty} 1 / k==0$, we can choose $N_{2}>N_{1}$ so that $\sum_{k=1}^{N_{i}} 1 / k>(2 M / c)+1$. Now choose $\delta$ such that $0<\delta<\log \left[1+\left(1 / N_{2}\right) \log N_{2}\right.$. Then for each $k \leq N_{2}$, we have

$$
h^{2}<h^{\operatorname{lig}\left(1+\left\{1 / N_{2}\right): \log N_{2}\right.} \leq 1+\left(1 / N_{2}\right) ;
$$

and if $1<s<1+\delta$

$$
(1 / k)-\left(1 / k^{s}\right)<\left(k^{\delta}-1\right) / k^{s}<k^{\delta}-1<1 / N_{2} .
$$

Summing from $k=1$ to $\mathrm{N}_{2}$, we obtain

$$
\begin{aligned}
\sum_{k=1}^{N_{N}}\left(\frac{1}{k^{s}}\right) & >\sum_{k=1}^{N_{k}}\left(\frac{1}{k}-\frac{1}{N_{2}}\right) \\
& >\left(\frac{2 M}{\epsilon}\right)+1-1 \\
& =\frac{2 M}{\epsilon} .
\end{aligned}
$$

Thus for $1<s<1+\delta$,

$$
\begin{aligned}
s(s) & >\sum_{k=1}^{N_{2}} \frac{1}{k^{s}} \\
& >\frac{2 M}{\epsilon} .
\end{aligned}
$$

and

$$
\begin{aligned}
\left|\frac{1}{s(s)} \sum_{k=1}^{\infty} \frac{x_{k}}{k^{v}}-L\right| & \leq \frac{1}{s(s)} \sum_{k=1}^{N_{1}} \frac{1}{k^{s}}\left|x_{k}-L\right|+\frac{1}{s(s)} \sum_{k>N_{1}} \frac{1}{k^{s}}\left|x_{k}-L\right| \\
& <\frac{\epsilon}{2 M} M+\frac{\epsilon}{2} \\
& =\epsilon .
\end{aligned}
$$

Hence,

$$
\lim _{s \rightarrow 1^{+}} \frac{1}{s(s)} \sum_{k=1}^{\infty}\left(\frac{x_{k}}{k^{s}}\right)=L
$$

Now assume $x$ is a real number sequence which diverges to $\infty$. Then for each number $M>0$ there exists a positive interger $N$ such that $x_{k}>M+1$ for all $k>N$. Suppose $s>1$ and consider

$$
\begin{aligned}
\frac{1}{s(s)} \sum_{k=1}^{\infty} \frac{x_{k}}{k^{s}} & >\frac{1}{s(s)} \sum_{k=1}^{N} \frac{x_{k}}{k^{s}}+\frac{M+1}{s(s)} \sum_{k>N} \frac{1}{k^{s}} \\
& =\frac{1}{\zeta(s)} \sum_{k \rightarrow 1}^{N}\left|\frac{x_{k}-1}{k}\right|+(M+1) .
\end{aligned}
$$


Since $\zeta(s) \rightarrow \infty$ as $s \rightarrow 1^{+}$, we see that if $s$ is sufficiently close to 1 on the right, then

$$
\left|\frac{1}{s ! s)} \sum_{k=1}^{N}\right| \frac{x_{k}-M-1}{k^{s}}||<1 ;
$$

this implies that

$$
\begin{aligned}
\frac{1}{s(s)} \sum_{k=1}^{\infty} \frac{x_{k}}{k} & >\frac{1}{s(s)} \sum_{k=1}^{N}\left(\frac{x_{k}-M-1}{k^{s}}\right)+M+1 \\
& >-1+M+1 \\
& =M .
\end{aligned}
$$

Since $M>0$ was chosen arbitrarily, we conclude that

$$
\lim _{s \rightarrow 1^{+}} \frac{1}{s(s)} \sum_{k=1}^{\infty} \frac{x_{k}}{k^{s}}=\infty
$$

A previous definition of "zeta summability" was given in Diaconis [2]. In that paper the bounded sequence $x$ is said to be zeta summable to $L$ if

$$
\lim _{s \rightarrow 1^{+}}(s-1) \sum_{i=1}^{\infty} \frac{x_{i}}{i^{s}}=L
$$

This is equivalent to the difinition of the zeta method introduced in this paper. There equivalence is an immediate consequence of the fact that $\lim _{s \rightarrow 1+} \zeta(s)(s-1)=1$.

Recall that a Stoltz domain of angle, where $0<\alpha<\pi / 2$, is a complex number set of the form

$$
S(\alpha)=\{w:|\operatorname{Arg}(w-1)|<\alpha, \text { and }|w|<1\} .
$$

(Powell et al [3]).

We shall use a variant of this concept, which we shall call a "reflected Stoltz domain of angle $\alpha$ "

$$
S^{*}(\alpha)=\left\{w:|\operatorname{Arg}(w-1)|<\alpha \text { and } R_{e}(w)>1\right\} .
$$

This concept is now used to extend the zeta method to one using a complex-valued function limit, and we establish the regularity of this extension.

THEOREM 2. Let $S^{*}(\alpha)$ be a reflected Stoltz domain of angle $\alpha$; if the sequence $x$ converges to $L$ then

$$
\lim _{w \rightarrow 1, v \in S^{*}(\alpha)} \frac{1}{S(w)} \sum_{k=1}^{\infty}\left(\frac{x_{k}}{k^{w}}\right)=L
$$

The proof of Theorem 2 that we shall give needs the following preliminary result.

LEMMA 1. For $w=\sigma+i t, w \in S^{*}(\alpha)$, and $w$ sufficiently close to 1 , we have

$$
\frac{1}{|s(w)|} \sum_{k=1}^{\infty}\left|\frac{1}{k^{w}}\right| \leq 2 \sec \alpha \text {. }
$$

Proof. Since $\zeta(w)$ can be expanded in the form $(w-1)^{-1}+P(w-1)$, where $P(w-1)$ is a power series in (w - 1), (Hardy [4], p. 333), we have 


$$
\begin{aligned}
& \frac{1}{|s(s)|} \sum_{k=1}^{\infty} \frac{1}{k^{w}}=\frac{|s(\sigma)|}{|s(w)|} \\
&=\frac{\left|\frac{1}{\sigma-1}+P(\sigma-1)\right|}{\left|\frac{1}{w-1}+P(w-1)\right|} \\
& \rightarrow\left|\frac{w-1}{\sigma-i}\right| \\
&
\end{aligned}
$$

Since the limit value $|w-1| /|\sigma-1| \leq \sec \alpha$ for $w \varepsilon S^{*}(\alpha)$, this proves the assertion.

Now we prove Theorem 2.

Proof (of Theorem 2). Let $\varepsilon>0$. Since $x$ converges to $L$, we can choose $N_{1} \ni \quad\left|x_{k}-L\right|<(\varepsilon / 4)$ $\cos \alpha$ for $k \geq N_{1}$. Let $\sum_{k=1}^{N_{1}}\left|x_{k}-L\right|=M$. Since $\zeta(w) \rightarrow \infty$ as $w \rightarrow 1$, we have $1 / \zeta(w)<\varepsilon / 2 M$ for $w$ sufficiently close to 1 .

Now for $w \in S^{*}(\alpha)$, we have

$$
\begin{aligned}
\left|\frac{1}{s(w)} \sum_{k=1}^{\infty} \frac{x_{k}}{k^{w}}-L\right| & \leq \frac{1}{|s(w)|} \sum_{k=1}^{\infty} \frac{1}{\left|k^{w}\right|} \cdot\left|x_{k}-L\right| \\
& =\frac{1}{|s(w)|}\left[\sum_{k=1}^{N_{1}} \frac{1}{\left|k^{w}\right|}\left|x_{k}-L\right|+\sum_{k>N_{1}} \frac{1}{\left|k^{w}\right|}\left|x_{k}-L\right|\right] \\
& <\frac{M}{\mid s(w) !}+\frac{1}{|s(w)|} \cdot \frac{c}{4} \cos a \sum_{k=1}^{\infty} \frac{1}{\left|k^{w}\right|} \\
& <\frac{t}{2}+\frac{\epsilon}{4}(\cos \alpha) 2 \sec \alpha \\
& =\epsilon .
\end{aligned}
$$

Next we prove a limitation theorem which asserts that the $\zeta$-summability method cannot sum a sequence that diverges too rapidly.

THEOREM 3. If a complex number sequence $x$ is $\zeta$-summable, then for each $s>1, x_{n}=0\left(n^{s}\right)$. Moreover, the term $\circ\left(n^{S}\right)$ is the best possible in the sense that the conclusion fails if $n^{S}$ is replaced by any real sequence to such that $t_{n} / n^{s}$ decreases to zero.

Proof. For $x$-to be $\zeta$-summable, $x$ must be in the domain of the $\zeta$-summability method. Therefore $\sum_{n=1}^{\infty}\left(x_{n} / n^{s}\right) \quad$ converges for all $s>1$, which implies that $\lim _{n}\left(x_{n} / n^{s}\right)=0$. If $n^{s}$ is replaced by $t_{n}$, where $t_{n} / n^{s}$ decreased to 0 , then we assert that it will not be true that $x_{n}=o\left(t_{n}\right)$ whenever $x$ is $\zeta$-summable. This is equivalent to showing that there is a sequence $x$ such that $x$ is $\zeta$-summable and $x_{n} \neq 0\left(t_{n}\right)$. Define the sequence $x$ by $x_{n}=(-1)^{n+1} t_{n}$, so that

$$
\sum_{n=1}^{\infty} \frac{x_{n}}{n^{s}}=\sum_{n=1}^{\infty}(-1)^{k+1} \frac{t_{n}}{n} .
$$

This is a convergent alternating series, and its (positive) sum is bounded by its first term $t_{1}$. 
Hence,

$$
\lim _{s \rightarrow 1^{+}} \frac{1}{s(s)} \sum_{n=1}^{\infty} \frac{x_{n}}{n^{s}}=0
$$

i.e., $x$ is $\zeta$-summable to 0 . But $x_{n} \neq 0\left(t_{n}\right)$ because for each $n,\left|x_{n} / t_{n}\right|=1$.

\section{ZETA SUMMABILITY MATRICES}

Definition. Let $t$ be a sequence of real numbers such that $t(n)>1$ for every $n$ and $\lim _{n} t(n)=1$.

Then the zeta matrix $z_{\mathrm{t}}=\left[z_{n k}\right]$ associated with the sequence $t$ is defined by

$$
\mathrm{z}_{\mathrm{nk}}=\frac{1}{s(\mathrm{t}(\mathrm{n})) \mathrm{k}^{\mathrm{l}(\mathrm{u})}} \quad \text { for } \mathrm{n}, \mathrm{k}=1,2,3, \ldots
$$

In this section we make use of two well-known theorems in summability theory, which we shall subsequently cite by name only; they are Silverman-Toeplitz Theorem ([5]and [6]) and the Knopp-Lorentz Theorem [7]. It is an easy calculation to show that $Z_{1}$ satisfies the conditious of the Silverman-Toeplitz Theorem for regularity. Moreover, $Z_{t}$ is totally regular because all of its entries are positive real numbers ([3] p. 35). We summarize these observations in the following theorem.

THEOREM 4. The zeta matrix $Z_{t}$ associated with the sequence $t$ is totally regular.

The next result is a characterization of those sequences $t$ for which $Z_{t}$ is an $l-l$ matrix, i.e., $Z_{t}$ maps $I_{1}$ into $/ 1$.

THEOREM 5. The matrix $Z_{t}$ is an $l-l$ matrix if and only if $t-l$ is in $l_{1}$.

Proof. Since each row sequence of the matrix $Z_{t}$ is decreasing, the set of the sums of column sequences of the matrix $Z_{t}$ is bounded by the sum of its first column entries. Therefore by the Knopp-Lorentz Theorem, it is enough to show that the first column sum is finite whenever $\sum_{u=1}^{\infty}$ $(t(n)-1)$ is convergent. This is a consequence of the inequality

$$
\sum_{n=1}^{\infty} \frac{1}{s(i(n))} \leq \sum_{n=1}^{\infty}(t(n)-1)
$$

which follows immediately from the fact that for $s>1$,

$$
\begin{aligned}
\frac{\mathrm{s}-1}{\mathrm{~s}} & \leq \frac{1}{\mathrm{~s}(\mathrm{~s})} \\
& \leq \mathrm{s}-1
\end{aligned}
$$

Hence $Z_{t}$ is an $\mathrm{H}$ matrix.

Conversely, assume $Z_{t}$ maps $l_{1}$ to $l_{1}$. Since $t(n)>1$ and $\lim n t(n)=1$ for every $n$, we can choose a positive integer $N$ such that $0<t(n)-1<1$ for $n \geq N$. Suppose $t-1$ is not in $/ 1$; then

$$
\begin{aligned}
\sum_{u=N}^{\infty}\left(\frac{1}{t(n)}\right) & =\sum_{n=-N}^{\infty}\left(\frac{t(n)-1}{t(n)}\right) \\
& >\sum_{n=-N}^{\infty}\left(\frac{t(n)-1}{2}\right) \\
& =\infty .
\end{aligned}
$$


Now $\beth_{n-1}^{\prime \prime}(1 / \zeta(t(n)))$ diverges to infinity because of the inequality $\left.1 / \zeta(t(n))\right) \geq(1-1 / t(n))$ as in $\left(^{*}\right)$. Therefore, by the Knopp-Lorentz Theorem, $Z_{t}$ is not an $l-l$ matrix. This completes the proof of the theorem.

4. INCLUSION THEOREMS.

In this section we compare the strength of the zeta method and the zeta matrix methods to several well-known summability methods. Throughout this section $C_{\alpha}$ denotes the Cesaro summability matrix of order $\alpha$ and $E_{r}$ the Euler-Knopp summability matrix of order $r$.

LEMMA 2. If $x$ is a sequence that is $C_{1}$-summable, then $x$ is in the domain of the $\zeta$-summability method, and hence, $x$ is in the domain of every $Z_{t}$ method.

Proof. Assume that $x$ is $C_{1}$-summable to $L: \lim _{n}\left(x_{1}+\ldots+x_{n}\right) / n=L$. To get the conclusion it is enough to show that the abscissa of convergence $\sigma_{0}$ of the Direchlet series $\sum_{n=1}^{\infty} x_{n} / n^{s}$ is less than or equal to 1 , where $\sigma_{\circ}$ is given by

$$
\sigma_{0}=\limsup _{\mathrm{u} \rightarrow \infty} \frac{\log \left|\sum_{k=1}^{u} x_{k}\right|}{\log \mathrm{n}} .
$$

(Hardy et al [8] or Titschmarch [9]). Since $x$ is $c_{1}$-summable to $L$, there exists a positive integer $N$ such that if $n \geq N$, then

$$
\frac{\left|\sum_{k=1}^{n} x_{k}\right|}{n} \leq|L|+1 .
$$

This implies that $\left|\sum_{k=1}^{n} x_{k}\right| \leq n(|L|+1)$, sc

$$
\log \left|\sum_{k=1}^{n} x_{k}\right| \leq \log [n(|L|+1)] .
$$

Therefore

$$
\begin{aligned}
& \sigma_{0}=\limsup _{n \rightarrow \infty} \frac{\log \left|\sum_{k=1}^{n} x_{k}\right|}{\log n} \\
& \leq \limsup _{n \rightarrow \infty}\left(\frac{\log n(|| L \mid+1)}{\log n}\right) \\
& =1 .
\end{aligned}
$$

THEOREM 6. The $Z_{t}$ method includes the $C_{1}$ method.

Proof. This inclusion is equivalent to the regularity of the matrix $Z_{t} C_{1}{ }^{-1}$, which can be verified by direct calculation using the Silverman-Toeplitz Theorem.

The following example shows that the $C_{1}$ method does not include the $Z_{t}$ method. 
EXAMPLE.

$$
\begin{aligned}
& \text { iset } x=\left\{(-1)^{k} k\right\} \text {; then } \\
& \left(i_{1}, x\right)_{n}=-\sum_{k=1}^{\infty} \frac{(-1)^{k} k}{(t(n)) k^{t(n)}} \\
& =\frac{1}{s(t(n))} \sum_{k=1}^{\infty} \frac{(-1)^{k}}{k^{t(n)-1}}
\end{aligned}
$$

Since

$$
\begin{aligned}
-1 & \leq \sum_{k=1}^{\infty} \frac{(\cdot-1)^{k}}{k^{t(n)-1}} \\
& \leq 0 \quad \text { for } t^{(u)}>1
\end{aligned}
$$

and $\lim _{n} \zeta(t(n))=\infty$, it is easy to see that $\lim _{n}\left(Z_{t} x\right)_{n}=0$. On the other hand, we have

$$
\begin{aligned}
\left(C_{1} \times\right)_{n} & =\frac{1}{n} \sum_{k=1}^{n}(-1)^{k} k \\
& =\left\{\begin{array}{l}
\frac{1}{2}, \text { if } n \text { is even } \\
\frac{-1}{2}, \text { if } n \text { is odd. }
\end{array}\right.
\end{aligned}
$$

Thus $\lim _{n}\left(c_{1} x\right)_{n}$ does not exist, so $x$ is not $C_{1}$-summable.

By a "continuous parameter sequence-to-function transformation", we mean a summablility method $F$ that is determined as follows by a fuction sequence $\left\{f_{k}(z)\right\} \sum_{k=1}^{\infty}$ : for a given sequence $x$ form the function

$$
F_{x}(z)=\sum_{k=1}^{\infty} f_{k}(z) x_{k} ;
$$

if $\lim _{\mathbf{Z}} \rightarrow \mathbf{a} F_{\mathbf{X}}(\mathbf{z})=L$, then we say that " $\mathrm{X}$ is $\mathrm{F}$-summable to $\mathrm{L}$ ". For a given function sequence $\left\{f_{k}(z)\right\}_{k \cdots 1}^{a}$ and a given number sequence $t$, we can also form an associated matrix $F_{t}$, which is given by

$$
F_{t}[n, k]=f_{k}(t(n))
$$

The next lemma, which will be used to compare the $C_{1}$ method and the $\zeta$ method, is a comparision of the method if and the associated matrix method $F_{t}$.

LEMMA 3. Let $F$ be a continuous parameter sequence-to-function transformation as in ("*) and define the sequence sets

$$
\begin{aligned}
& s_{r}=\left\{x: \lim _{z: n} F_{x}(z) \text { exisls }\right\} . \\
& S_{F_{t}}=\left\{x: F_{t} x \text { is convergent }\right\},
\end{aligned}
$$

and

$$
\mathrm{T}=\left\{\mathrm{t}: \lim _{\mathrm{u}} \mathrm{u}(\mathrm{u})=\mathbf{a}\right\}
$$


then

$$
S_{F}=\bigcap_{1 \cdot T} S_{F_{1}}
$$

Proof. We show that each of $S_{F}$ and $\bigcap_{t \in T} S_{F_{t}}$ contains the other. Since $F_{t}$ includes $F$ for $t$ in $T$, we have

$$
S_{F} \subseteq \bigcap_{t \in T} S_{F_{t}}
$$

To prove the reverse inclusion, we consider a sequence $x$ which is not in $S_{F}$. It follows that $\lim _{Z} \rightarrow a F_{X}(z)$ does not exist. By the sequential criterion for function limits (Almsted [10], p. 73), there is a sequence $t$ ) in $T$ such that $\lim _{n}\left(F_{t} x\right)_{n}$ does not exist. This implies that $x$ is not in the set $S_{F_{t}}$. Hence $x$ is not in the set $r \cdot e^{\prime} \mathrm{T}_{\mathrm{F}}$.

THEOREM 7. The $\zeta$-summability method is stronger than the $C_{1}$ method.

Proof. By Lemma 3, we have $S_{\zeta}=\Gamma_{t, T} S_{z_{t}}$. Since the $Z_{t}$ method includes the $C_{1}$ method for all $t$ in $T$, we have $S_{C_{1}} S i$ ? te $S_{z_{t}}=S_{c}$. Now if $x$ is a sequence that is $C_{1}$-summable to $L$, then $x$ is $Z_{t}$ summable to $L$ for all $t$ in $T$. Therefore the sequential criterion for function limits ensures that $x$ is $\zeta$-summable to $L$. Hence, the $\zeta$ method includes the $C_{1}$ method. It is easy to see that the $C_{1}$ method does not include the $\zeta$ method because $C_{1}$ method does not include the $Z_{t}$ method.

As a consequence of Theorem 6 , we can infer that $Z_{t}$ includes any method that is included by $C_{1}$. For example, $Z_{t}$ includes the divisor method $D_{r}$ for $r>0$. (Fridy [11]).

Let $\mathrm{H}_{2}$ denote the Holder method of order 2. By arguing as in the proof of Theorem 6, we can prove

THEOREM 8. If the sequence $x$ is $H_{2}$-summable to $L$ and $x$ is in the domain of the $Z_{t}$ method, then $x$ is $Z_{t}$ summable to $L$.

COROLLARY. If the sequence $x$ is $H_{2}$-summable to $L$ and $x$ is in the domain of the $\zeta$-summability method, then $x$ is $\zeta$-summable to $L$.

The conclusion of the preceding Corollary does not hold if $x$ is not in the domain of the $\zeta$ method. This is shown by the following example.

EXAMPLE. Let $\mathrm{x}$ be the sequence defined by

$$
x_{n}=:=\left\{\begin{array}{l}
(-1)^{k} k^{\frac{3}{2}}, \text { if } n=2 k, k=1,2, \ldots \\
(-1)^{k+1} k^{\frac{3}{2}}, \text { if } n=2 k-1, k=1,2, \ldots,
\end{array}\right.
$$

If $x \leq 3 / 2$, then the series $\sum_{n=1}^{\infty}\left(x_{n} / n^{5}\right)$ is divergent because its $n^{\text {th }}$ term does not approach 0 . Therefore $x$ is not in the domain of the $\zeta$ method, and hence, $x$ is not $\zeta$-summable. Now we show that $x$ is $H_{2}$-summable to zero. Since $\left(C_{1} x\right)_{2 k-1}=(-1)^{k+1} k^{3 / 2} /(2 k-1)$ and $\left(C_{1} x\right)_{2 k}=0$, we see that the (odd) partial sums alternate in sign after $k=3$; thus the partial sum is not greater than the last term, which is $0\left(k^{1 / 2}\right)$. 
Therefore, upon dividing by $2 k-1$ to form $C_{1}\left(C_{1} x\right)_{2 k-1}$, we have

$$
\begin{aligned}
\left(H_{2} x\right)_{2 k-1} & =\left(\frac{1}{2 k-1}\right) \circlearrowleft\left(k^{\frac{1}{2}}\right) \\
& =O\left(k^{-\frac{1}{2}}\right) \\
& =o(1) .
\end{aligned}
$$

which proves that $\mathrm{x}$ is $\mathrm{H}_{2}$-summable to zero.

Since the Holder method of order 2 is equivalent to the Cesaro method of order 2 (Hardy [4], $p$ 103), we can immediately get the following theorem.

THEOREM 9. If $x$ is a sequence which is $C_{2}$-summable to $L$ and $x$ is in the domain of the summability method, then $\mathrm{x}$ is $\zeta$-summable to $\mathrm{L}$.

It is well known that for each number $r$ satisfying $0<r<1$ and any nonzero real number $\alpha, E_{r}$. By using these facts, we have the following result.

THEOREM 10. The $\zeta$ method is not included in $E_{r}$ for $0<r<1$.

The following example shows that the $\zeta$ method does not include $E_{r}$ for $0<r<1$.

EXAMPLE. Given $r$ between 0 and 1 choose $\varepsilon>0$ satisfying $r<2 /(2+\varepsilon)$. Next define $x_{k}=(-1-\varepsilon)^{k}$. Then

$$
\begin{aligned}
\left(\mathrm{E}_{\mathrm{r}} \mathrm{x}\right)_{\mathrm{u}} & =\sum_{r=0}^{\mathrm{n}}(\mathrm{k}) \mathrm{r}^{\mathrm{k}}(1-\mathrm{r})^{\mathrm{r}-\mathrm{k}}(-1-\epsilon)^{\mathrm{k}} \\
& =[(-1-\epsilon) \mathrm{r}+(1-\mathrm{r})]^{\mathrm{u}} \\
& =[(-2-\mathrm{c}) \mathrm{r}+1]^{\mathrm{u}} .
\end{aligned}
$$

Since $0<r<2 /(2+\varepsilon)$, we have $-1<(-2-\varepsilon) r+1<1$. This implies that

$$
\begin{aligned}
\lim _{\mathbf{n}}\left(E_{\mathrm{r}} \mathrm{x}\right)_{\mathrm{u}} & =\lim _{\mathbf{n}}[(-2-c) \mathrm{r}+1]^{\mathrm{n}} \\
& =0,
\end{aligned}
$$

i.e., $x$ is $E_{r}$ summable to 0 . But $x$ is not in the domain of the $\zeta$ method because the series

$$
\sum_{k=1}^{\infty} \frac{(-1-\epsilon)^{k}}{k^{s}}
$$

is not convergent for any $\mathrm{s}$, whence $\mathrm{x}$ is not in the domain of the $\zeta$ method.

ACKNOWLEDGEMENT. This work is a portion of the author's doctoral dissertation, written under the supervision of Professor J. A. Fridy at Kent State University, Kent, OH, 1985.

\section{REFERENCES}

1. TITSCHMARCH, E.C. The Theory of the Riemann Zeta Functions, Oxford University Press, London, 1951.

2. DIACONIS, P. Examples for the Theory of Infinite Iteration of Summability Methods, Canadian Journal of Mathematics, Vol. XXIX, No. 3, 1977, pp. 489-497. 
3. POWELL, R.E. and SHAH, S. M. Summability Theory and Applications, Van Nostrand, London, 1972

4. HARDY, G. H. Divergent Series, Oxford University Press, London, 1949.

5 SILVERMAN, L.L. On the Definition of the Sum of a Divergent Series, University of Missouri Studies, Mathematical Series, No. 1, 1913, pp. 1-96.

6. TOEPLITZ, O. Über allgenmeine linear Mittelbrildungen, Prace Matematyzns-fizyczne (Warsaw), No. 22,1911 , pp. 113-119.

7. KNOPP, K. and LORENTZ, G.G. Beiträge zur absoluten Limitierung, Arch. Math., No. 2, 1949, pp. 10-16.

8. HARDY, G.H. and RIESZ, M. The General Theery of Dirichlet's Series, Cambridge Tracts in Mathematics and Mathematical Physics, No. 18, Cambridge University Press, 1915.

9. TITSCHMARCH, E.C. The Theory of Functions, Lowe and Brydone, Printers, London, 1949.

10. OLMSTED, M.H. Advanced Calculus, Appleton-Century-Crofts, New York, 1961.

11. FRIDY, J.A. Divisor Summability Methods, Journal of Mathematical Analysis and Applications, Vol. 12, No. 2, 1965, pp. 235-243. 


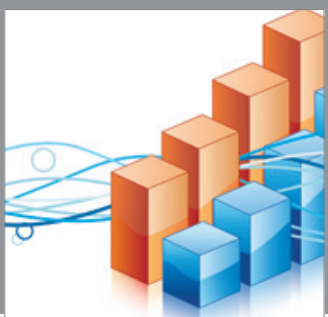

Advances in

Operations Research

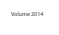

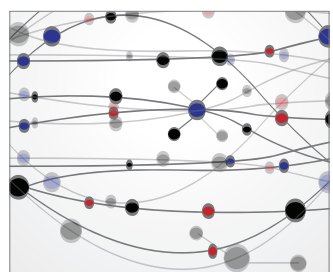

\section{The Scientific} World Journal
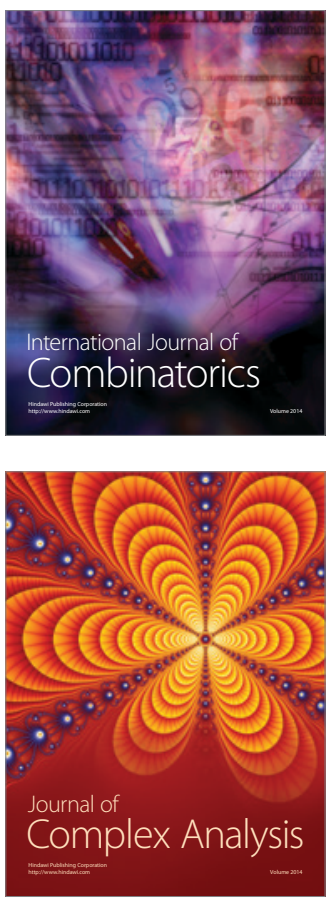

International Journal of

Mathematics and

Mathematical

Sciences
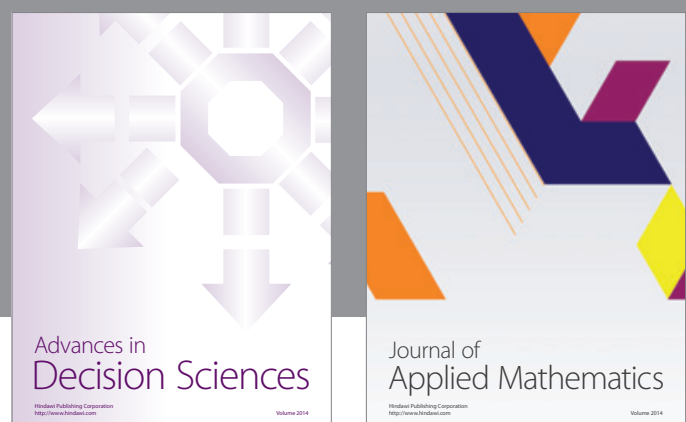

Journal of

Applied Mathematics
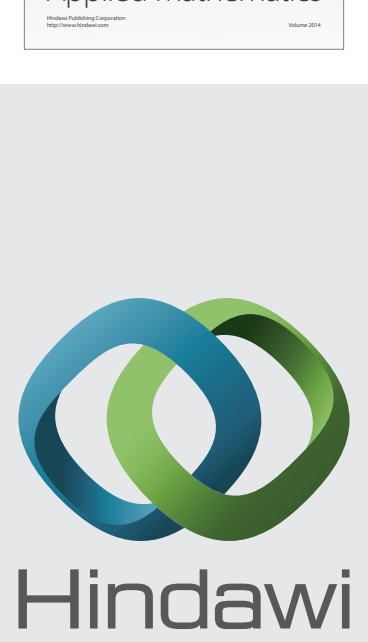

Submit your manuscripts at http://www.hindawi.com
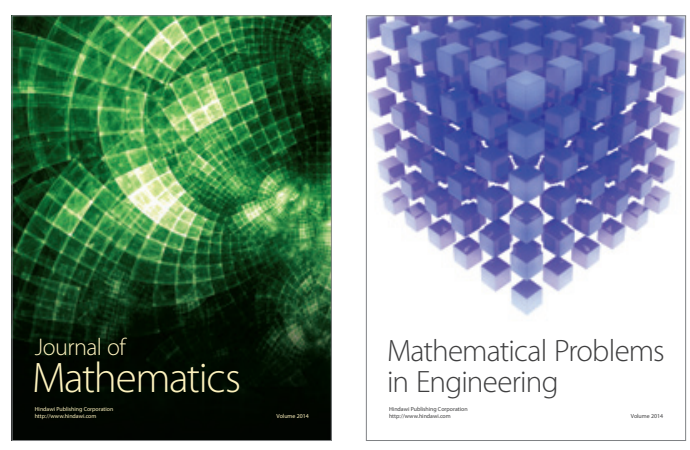

Mathematical Problems in Engineering
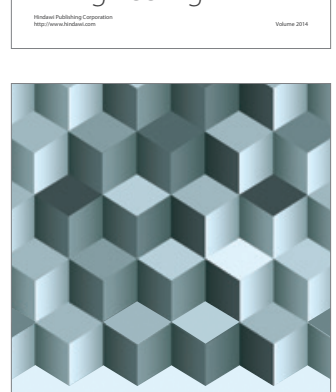

Journal of

Function Spaces
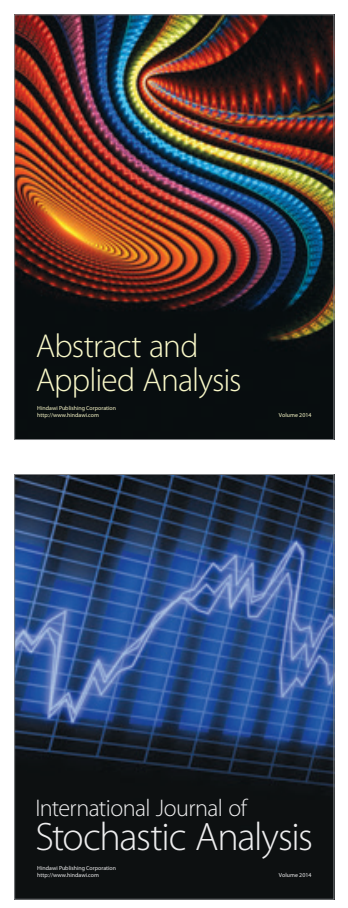

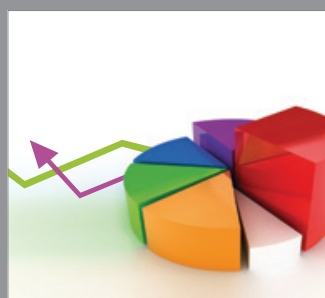

ournal of

Probability and Statistics

Promensencen
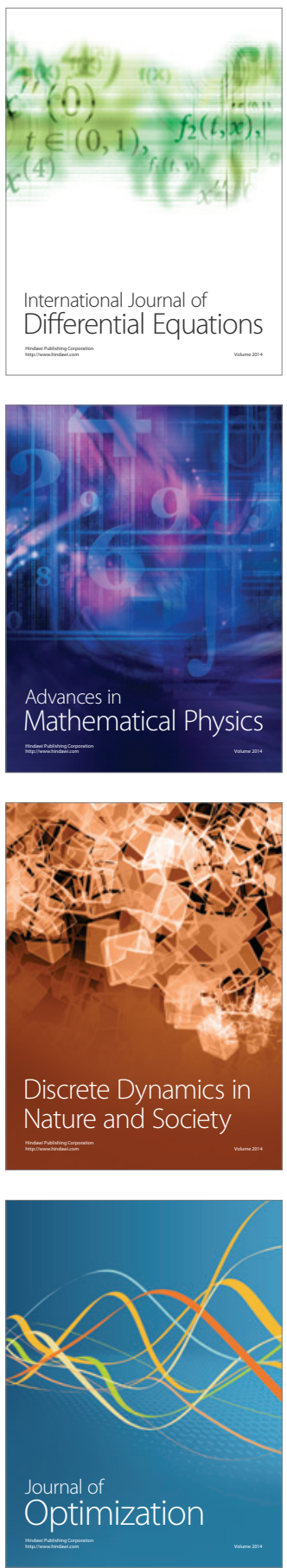